Pacific

Journal of

Mathematics

SINGULARITIES OF THE PROJECTIONS OF SURFACES IN 4-SPACE

Vera Carrara, J. Scott Carter, and Masahico Saito 


\title{
SINGULARITIES OF THE PROJECTIONS OF SURFACES IN 4-SPACE
}

\author{
Vera Carrara, J. Scott Carter, and Masahico Saito
}

We study the singularities of maps of surfaces from a knot theoretic point of view. We define and study colors and signs of branch and triple points on knotted surface projections and give formulas among the numbers of these. We prove that cusps can be canceled on the planar projections of knotted surfaces. For orientable knotted surfaces, we prove that both cusps and branch points can be canceled.

\section{Introduction.}

In classical knot theory (knotted circles in 3-space), it is common to use the diagram and the projection of a knot to perform calculations. For example, the Jones [19] and Alexander-Conway [16] polynomials can be computed by a skein relation. Vassiliev [29] invariants obey similar skein relations [2] for singular knots. The combinatorics contained in the projection are used extensively in [23] and [25] for example, and the algebraic information that is encoded in either a diagram or a projection can be found in [3].

In this paper, we explore projections of knotted surfaces in 4-space and their isotopies. By a knotted surface we mean a smoothly embedded surface in 4-dimensional Euclidean space and we work in the smooth category. We define and relate signs of triple points of the projection of surfaces and relate these notions to the normal Euler number. There are two ways of defining signs for triple points. In the first way, oriented surfaces are considered, and the sign is the orientation of the normals to the top, middle, and bottom sheets, in that order. In the second way, the surface need not be oriented, but a checker-board shading is initially chosen. Then normals to the top, middle, and bottom sheets are chosen to point into a black region. These two notions of signs are related, and formulas relating their numbers will be given. Such numbers are interelated to the number of signed and colored branch points when these are present. It is, then, natural to consider the numbers of other singularities on knotted surface projections. We show that a knotted surface can be isotoped so that the planar projection has one or no cusps. For orientable knotted surfaces, we show that both cusps and branch points can be canceled. 
Acknowledgements. JSC gratefully acknowledges support from FAPESP, NSA, and the University of South Alabama Support and Development Fund when the research was being conducted. The hospitality of IME-USP was appreciated. MS was supported, in part, by the University of South Florida Research and Creative Scholarship Grant Program under Grant Number 1249932RO. We had useful conversations with Maria A. Ruas while working on this project. The referee had a number of good comments that improved the exposition of this paper.

\section{Preliminaries.}

In this section we review knotted surface diagrams and surface braid theory that will be used in the paper. More details can be found in [15].

2.1. Generic projections and diagrams. For classical knots and links, under-arcs are broken in the projections to define knot diagrams. We generalize this notion to knotted surfaces as broken surface diagrams. First we develop some notation. Let $f: F \rightarrow \mathbf{R}^{4}$ denote a smooth embedding of a closed surface $F$ into 4-dimensional space (with the standard smooth structure). Choose a direction $v \in \mathbf{R}^{4}$ and project $\mathbf{R}^{4}$ onto a 3 -plane perpendicular to the vector $v$. Choose such an affine hyperplane so that $f: F \rightarrow \mathbf{R}^{4}$ lies in one component of $\mathbf{R}^{4} \backslash \mathbf{R}^{3}$. Denote such a projection by $p_{v}$ or $p$.

The embedding $f$ may be moved slightly so that the composition $p_{v} \circ f$ will be a generic surface in 3-space. In this case, any point of the image surface has a neighborhood $U$ in 3-space such that $p_{v}(f(F)) \cap U$ looks like one of the pictures indicated in Figure 1 on the left. In other words, there is a diffeomorphism of $U$ into $\mathbf{R}^{3}$ such that the image of $F$ coincides with the intersection of 1, 2 (a double point curve), or 3 coordinate planes (a triple point), or is like the cone on a figure 8 ( a branch point). The neighborhood of a double point curve, a triple point, a branch point are depicted in Figure 1 (A), (B), and (C) respectively, on the left of the figures.

There is an immersion in $\mathbf{R}^{3}$ of a compact 1-manifold with boundary whose image is the closure of the set

$$
\begin{aligned}
\left\{x \in p(f(F)) \mid p\left(f\left(x_{1}\right)\right)=x=\right. & p\left(f\left(x_{2}\right)\right) \\
& \left.\quad \text { for some } x_{1} \neq x_{2}, \quad \text { where } x_{1}, x_{2} \in F\right\} .
\end{aligned}
$$

The closure of the above set is called the double point set. The image of the boundary points of the 1-manifold are the branch points of the map $p \circ f$. At a triple point three curves meet transversely, so a triple point is a 6 -valent vertex.

Similarly, there is a closed 1-manifold (called the double decker manifold) which is immersed into $F$ such that the image (called the double decker set) is the closure of the set

$$
\left\{x \in F: \#(p \circ f)^{-1}(p(f(F))) \geq 2\right\} .
$$




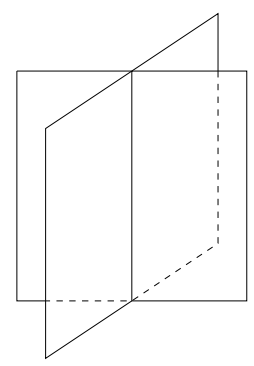

(A)
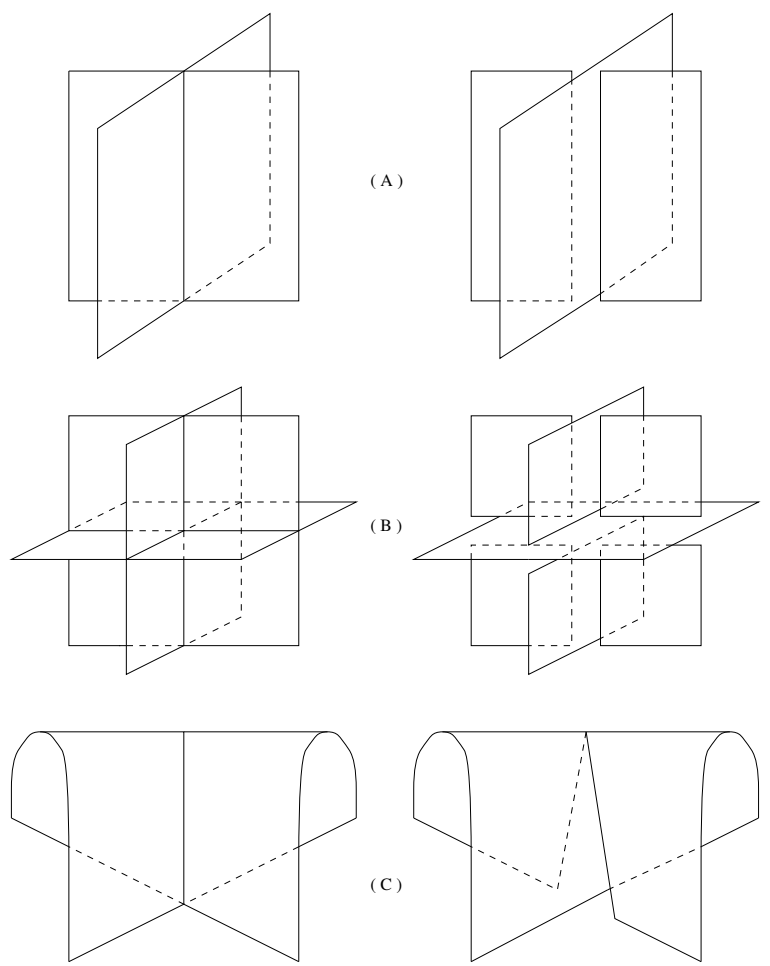

Figure 1. Projections and diagrams of knotted surfaces.

The portion of the double decker set on $f(F)$ that is most distant from the hyperplane of projection is called the lower-deck; the upper-deck is the portion of the double decker set that is closest to this hyperplane.

In a broken surface diagram of a knotted surface, the portion of the surface that is most distant from the hyperplane of projection is broken. We remove a small open tubular neighborhood of the lower-deck and embed the resulting surface with boundary into 3-space using the restriction of $p \circ f$. In a neighborhood of the branch points, the deleted neighborhood comes to a cone at each branch point. Then we can reconstruct an embedding in 4-space that is isotopic to the given knot $f(F)$, by gluing the neighborhood of the lower-deck to the embedded surface in 3-space: The boundary of this neighborhood lies in 3-space, and the interior protrudes "below" this hyperplane - in other words, the neighborhood lies in the same component of $\mathbf{R}^{4} \backslash \mathbf{R}^{3}$ that $f(F)$ does.

This broken surface diagram is the diagram of the knotting $f(F)$. Along open arcs of double points there are top and bottom sheets, and these are neighborhoods of the upper and lower deck (Figure 1 (A) right). In a neighborhood of a triple point, there is a top, middle, and bottom sheet (Figure 1 
(B) right), and a branch point is a point along a double curve at which the bottom sheet converges to the top sheet (Figure 1 (C) right).

In the discussion of Section 7, we consider the further projection of the diagram into a plane. In such a plane, the surface has fold lines and cusps. However, the definition of a knotted surface diagram does not include fold and cusp information.

2.2. Cusps and folds. Recall that a generic smooth map from a surface (2-dimensional manifold) to the plane has fold lines and isolated cusp points as its singularities. This fact was proved by Whitney (see [18] p. 146, and Fig. 8). In Fig. 8 surfaces are depicted in 3-space for visualization though the maps discussed here are from (abstract) surfaces to the plane. We call the image of the singularities in the plane (resp. the preimage in the surface) the fold curves and cusp points (resp. fold loci and cusp loci) to be precise. However, we call both fold curves (or folds) and cusp points (or cusps) when the context is clear.

2.3. Surface braid theory. The notion of surface braids, a generalization of Artin's braid theory, was originally proposed by Viro, and a similar notion had been used by Rudolph [28]. Recently the theory has been developed by Kamada $[\mathbf{2 1}, \mathbf{2 2}]$ and two of the authors $[\mathbf{9}, \mathbf{8}]$. We now review this theory.

Figure 2 shows how to express certain generic surfaces in 3 -space by means of planar graphs. We consider a surface $S$ (this $S$ corresponds to $f(F)$ in the preceding section) in a box $B=I_{1} \times I_{2} \times I_{3} \subset \mathbf{R}^{3}$ a schematic of which is depicted on the right of Figure 2, where $I_{j}$ denotes a copy of the unit interval, for $j=1,2,3$. We require that the surface $S$ in $B$ satisfies the following conditions.

- $S$ is generic.

- The boundary $\partial S$ of $S$ is contained in $\partial\left(I_{1} \times I_{2}\right) \times I_{3}$.

- The projection $\pi: I_{1} \times I_{2} \times I_{3} \rightarrow I_{1} \times I_{2}$ restricted to $S$ is a branched covering such that each branch point is of degree 2 .

Let $D$ be the double point set of $S$. Then $\pi(D) \subset I_{1} \times I_{2}$ is a planar graph. Let us call this an un-oriented chart. An un-oriented chart has univalent vertices corresponding to branch points, 4-valent vertices that correspond to the crossings of the projections of double arcs, and 6 -valent vertices corresponding to triple points of $S$. A generic intersection $S \cap I_{1} \times\{t\} \times I_{3}$, consists of intersecting strings in $I_{1} \times\{t\} \times I_{3}$. Such intersections are shown in the figure by movie strips.

By capping off the nested boundary circles on the boundary of the box $B$ by nested disjoint disks, we get a closed surface in 3 -space. If it is a projection of a knotted surface, taking such a closure gives rise to a closed knotted surface in 4-space. The generalization of Alexander's Theorem we need in this paper is as follows. 

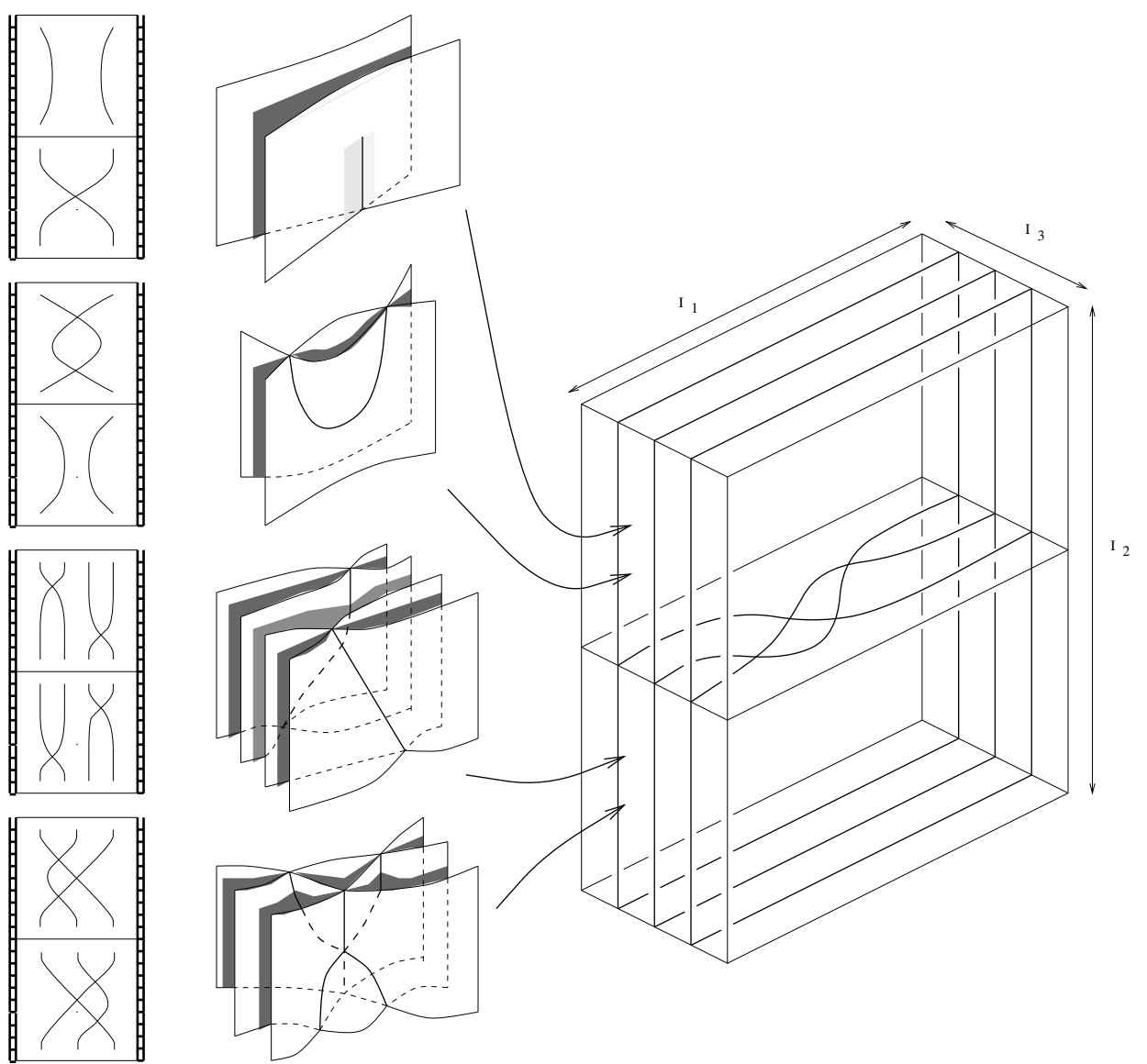

Figure 2. Generic surfaces, movies, and surface braids.

Theorem 2.3.1 (Viro, Kamada [22]). Any orientable closed knotted surface in 4-space can be isotoped to a closed surface braid.

\section{Colors and Signs of Multiple Point Sets.}

In this section we define signs and colors of double point and triple points, and study their combinatorics. Signs of triple points are defined in [13] and [14] in different ways. The former used the checker board coloring of regions, and the latter used the orientations. Furthermore in [6] the signs of branch points that were originally defined by Banchoff [1] were studied. In this section we combine these ideas and study their relationships.

Let $f: F \rightarrow \mathbf{R}^{4}$ be a knotted oriented surface. Let $p: \mathbf{R}^{4} \rightarrow \mathbf{R}^{3}$ be a projection such that $p \circ f$ is generic. Fix the orientation of $\mathbf{R}^{3}$ by the right hand rule. Define the normal vector $n$ at every point of $f(F)$ in $\mathbf{R}^{3}$ except at 
branch points such that the orientation of $\mathbf{R}^{3}$ matches the triple $\left(n, v_{1}, v_{2}\right)$ where $\left(v_{1}, v_{2}\right)$, tangent vectors to $f(F)$, form the orientation of $F$.

There is a checker-board coloring for the domains divided by $(p \circ f)(F)$ such that each domain is colored either black or white and adjacent regions (that is, regions sharing the same face) have different colorings. Fix such a coloring. Observe that if the other coloring is used, then the discussion below remains true with the words "white" and "black" and related numbers universally interchanged.

Definitions 3.1. 1. A distinguished region of $\mathbf{R}^{3} \backslash f(F)$ at a double point curve is one of four regions near the double point curve into which both the normal vectors point. A distinguished region of $\mathbf{R}^{3} \backslash f(F)$ at a triple point is one of eight regions near the triple point into which all the normal vectors point. Distinguished edges of the double point curves near a triple point are the three (among six) edges sharing the triple point that are contained in the closure of the distinguished region. (The last two of the above are defined in [14].)

2. (cf. [14]) The orientation of a double curve $\gamma$ is required to satisfy that the triple $\left(\gamma, v_{1}, v_{2}\right)$ matches the orientation of $\mathbf{R}^{3}$, where $v_{1}$ (resp. $v_{2}$ ) is a normal to the upper (resp. lower) sheet.

3. The color of the double point curves is defined to be the color of the distinguished region at the given double point curve.

4. The color of triple points is defined to be the color of the distinguished region at the given triple point.

5. The sign of a triple point is defined as follows. If the triple $\left(v_{1}, v_{2}, v_{3}\right)$ matches the right-hand orientation of $\mathbf{R}^{3}$, then the sign is positive and negative if otherwise. Here $v_{1}$ (resp. $v_{2}, v_{3}$ ) is a normal to the top (resp. middle, bottom) sheet at the given triple point.

6. (cf. [13]) A neighborhood of a branch point looks like the cone on the figure eight. The color of a branch point is defined to be the color of inside the figure eight.

The sign of a branch point is defined as follows. If the the double curve ending at the given branch point is oriented towards (resp. outwards) the branch point, then the sign is negative (resp. positive).

Figure 3 depicts some of the above definitions. In this figure, the normal vectors are depicted by $v_{i}, i=1,2,3$. The orientations of double point curves are also depicted in the right of the figure.

In this figure we observe that (1) the triple point is negative and black, (2) all three distinguished edges are black and exactly two of them point towards the triple point.

Indeed we have the following from [14]: The number of the distinguished edges whose orientations point towards the triple point is exactly one, or exactly two. In the figure the edges oriented towards the triple points are 

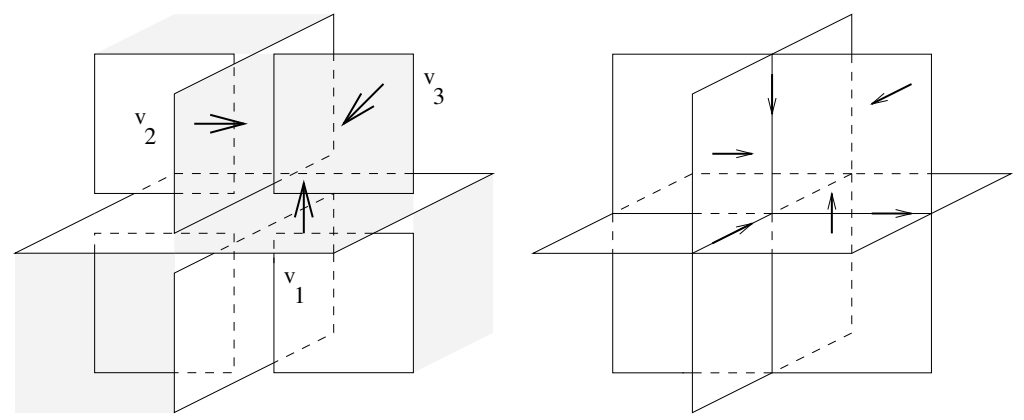

Figure 3. Orientations of double curves and colors of triple points.

that formed by top and middle sheets, and that formed by middle and bottom sheets. It is always the case that the colors of all distinguished edges are the same as the color of triple point, and the rest have the opposite color.

Proposition 3.2. The following hold at every triple point.

(1) All distinguished edges have the same color, this is the same as the color of the triple point, and the other three edges have the opposite color.

(2) The triple point is positive if and only if among the three distinguished edges the edge formed by the top and bottom sheets has its orientation pointing towards the triple point.

Proof. We check all the possible cases near triple points. First we can pick arbitrary normal vectors to the bottom and the middle sheet and move the given local picture to the one depicted in Fig. 3 so that the crossing information and normals match. Thus we have two choices for the normal to the top sheet, one depicted in the figure, and the other case. We now check the case where the normal to the top sheet is reversed. In such a case we observe: (1) the triple point is positive and white, (2) exactly one distinguished edge is oriented towards the triple point. This edge is formed by the top and the bottom sheet.

Next we check different coloring possibilities. There are the other coloring choices for both of the above two cases. For the opposite coloring of Fig. 3, we have (1) the triple point is negative and white, (2) exactly two distinguished edges are oriented towards the triple point. For the second case with the opposite coloring, we have (1) the triple point is positive and black, (2) exactly one distinguished edge is oriented towards the triple point.

This completes the proof.

Definition 3.3. We use the following notation for the numbers of various types of triple points and branch points. 


\begin{tabular}{||l||c|c|c|c||}
\hline Singularity \color-sign & black + & black - & white + & white - \\
\hline \hline Triple point & $T(B,+)$ & $T(B,-)$ & $T(W,+)$ & $T(W,-)$ \\
\hline Branch point & $P(B,+)$ & $P(B,-)$ & $P(W,+)$ & $P(W,-)$ \\
\hline
\end{tabular}

Here $P$ stands for a pinch point, another name of a branch point (to avoid the confusion between $\mathrm{b}$ for branch or black). Thus $T(B,+)$ denotes the number of black triple points that have a positive sign, etc.

\section{Formulas Coming from Normal Euler Number.}

The normal Euler number of an orientable knotted surface vanishes [17]. On the other hand, coloring was used in [13] to get a formula for normal Euler numbers for (not necessarily orientable) knotted surfaces. Here we apply the ideas of $[\mathbf{1 3}]$ in the orientable case to get new triple point formulas.

In [13] a sign for triple points was defined for not necessarily orientable surfaces that we call the colored sign of triple points. We review the definition here. There are eight regions near a triple point. Pick a black region, $B$, among four of them. Choose $\vec{v}_{1}, \vec{v}_{2}$ and $\vec{v}_{3}$ to be the normals to the top, middle, and bottom sheets, respectively such that these vectors point into the black region, $B$. See Figure 3. Define the triple point to have a positive colored sign if the orientation of 3-space defined by the ordered triple $\left(\vec{v}_{1}, \vec{v}_{2}, \vec{v}_{3}\right)$ matches the originally fixed orientation of the 3 -space. The colored sign is negative otherwise. Figure 3 depicts such vectors and checkerboard coloring at the neighborhood of a triple point. In the right hand convention this is a negative triple point. This definition depends upon the fixed orientation of the 3 -space and the coloring, but it does not depend upon the choice of the black region. Thus each triple point has a well-defined colored sign.

Now we relate the colored sign and the sign defined in Section 3.

Now suppose that the given surface is orientable (so that the signs of Section 3 are defined). If a triple point is (black, positive), then all the normals point into a black region, and they match the orientation of the space. Thus it has a positive colored sign. If it is (black, negative), it has a negative colored sign. If the triple point is (white, positive), then change the normal to the top sheet to get the triple $\left(-v_{1}, v_{2}, v_{3}\right)$ all of which point into a black region. They do not match the space orientation, so that the triple point has a negative colored sign. If the triple point is (white, negative), then for the same reason it is has a positive colored sign.

Thus we obtain:

Lemma 4.1. A triple point has a positive colored sign if it is either (black, positive) or (white, negative). A triple point has a negative colored sign if it is either (black, negative) or (white, positive). 
The convention on the colors and signs of branch points are the same (possibly up to multiplication by $(-1)$ for the sign, depending on the orientation convention). Thus Theorem 3.1 in [13] implies:

Proposition 4.2. It holds that

$$
\begin{aligned}
(T(B,+)+T(W,-)-T(B,-)-T(W,+)) & \\
& +(P(W,+)-P(W,-))+2(P(B,+)-P(B,-))=0 .
\end{aligned}
$$

Corollary 4.3. If the projection of the knotted surface is an immersion, then

$$
T(B,+)+T(W,-)-T(B,-)-T(W,+)=0 .
$$

\section{Colors and Signs for Surface Braids.}

In this section we describe signs that we defined in terms of surface braid theory.

5.1. Conventions. We choose the plane of projection for a surface braid so that the normal to the surface always points to the plane into which the braid chart is projected. We fix the checker-board coloring of 3 -space so that the region that contains infinity is black.

Then the region of 3 -space that lies between the sheet labeled $2 i-1$ and that labeled $2 i$ is white and the region between the sheet labeled $2 i$ and that labeled $2 i+1$ is black. Thus in a surface braid a double curve that is labeled by an odd (resp. even) number on the chart is black (resp. white).

On a braid chart, a triple point is a six-valent vertex labeled with $i$ and $i+1$. The edges labeled $i+1$ are distinguished edges. Proposition 3.2 implies (1) a triple point is black (resp. white) if $i+1$ is odd (resp. even); (2) a triple point is positive (resp. negative) if exactly one edge (resp. two edges) among those labeled $i+1$ is (resp. are) oriented towards the triple point. Call $i+1$ the label of the triple point.

A branch point is black (resp. white) if the edge is labeled by an odd (resp. even) number.

In summary we have:

Proposition 5.2. For braid charts the colors and signs are determined as follows.

(1) An edge is black if and only if it is labeled by an odd number.

(2) A triple point is black if and only if it is labeled by an odd number.

(3) A triple point is positive if and only if there is exactly one edge with the largest label oriented towards the triple point.

(4) A branch point is black if and only if the edge is labeled by an odd number.

Roughly, black if and only if odd. 


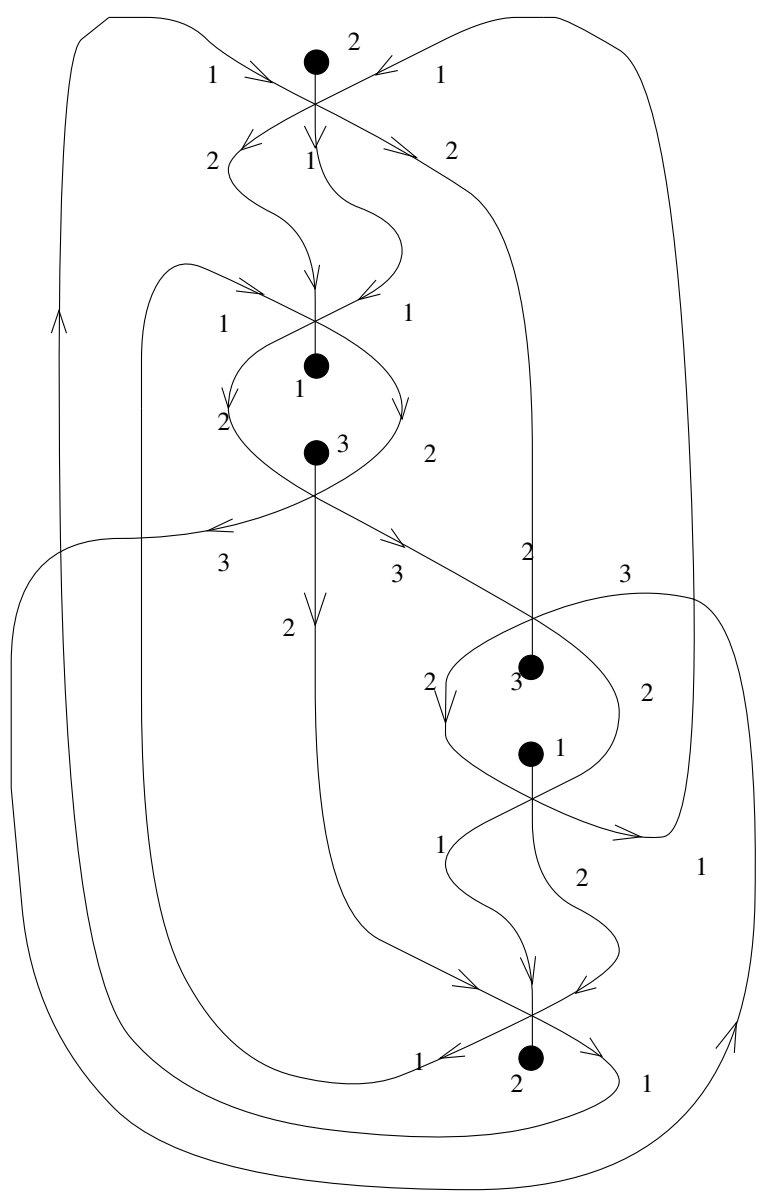

Figure 4. Braid chart of the 2-twist spun trefoil.

Example 5.3. A chart for the 2-twist spun trefoil knot was given in [20]. We reproduce this illustration in Fig. 4. The corresponding projection has 6 triple points and 6 branch points. By the above Proposition, we compute the numbers of colored and signed multiple points as follows.

\begin{tabular}{||l||c|c|c|c||}
\hline type \color - sign & black + & black - & white + & white - \\
\hline \hline Branch points & 2 & 2 & 1 & 1 \\
\hline Triple points & 1 & 1 & 2 & 2 \\
\hline
\end{tabular}

\section{Formulas for Colored and Signed Triple Points.}

In this section, we use the technique of smoothing that is depicted in Fig. 5 to establish some triple point formulas. 


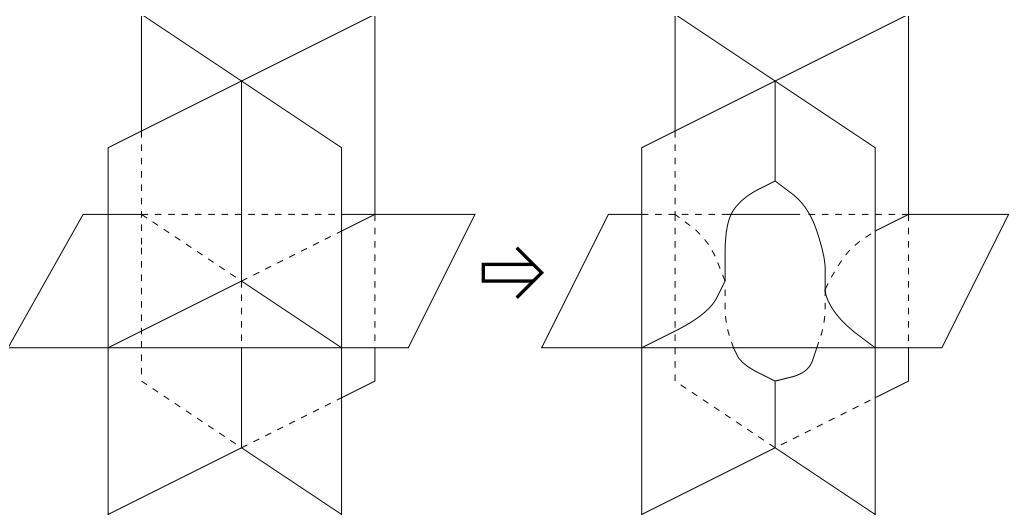

Figure 5. Smoothing a triple point.

Theorem 6.1. For the diagram of a knotted orientable surface it holds that

$$
\begin{array}{r}
T(B,+)-T(B,-)-T(W,+)+T(W,-)-P(W,+)+P(W,-)=0 \\
P(B,+)-P(B,-)+P(W,+)-P(W,-)=0 .
\end{array}
$$

Proof. We show that the following expression is zero.

$$
\begin{aligned}
T P(G) & =x(T(B,+)-T(B,-)-T(W,+)+T(W,-)) \\
& +z(P(B,+)-P(B,-))+(z-x)(P(W,+)-P(W,-)) .
\end{aligned}
$$

The smoothing of a triple point that is depicted in Fig. 5 is a 1-handle addition to the surface. It is realized by an embedding when the handle is attached between the top and middle sheets or the middle and bottom sheets. In other words after the smoothing we obtain a knotted surface diagram for a knotted surface in 4-space. Furthermore we can and do perform smoothings so that the result is orientable. This smoothing was used in $[6,12,11]$.

The smoothing replaces a (black, negative) triple point by a pair of a (black, negative) and a (white, positive) branch points. Similarly all the other replacements are a (black, positive) triple point by a pair of a (black, positive) and a (white, negative) branch points, a (white, positive) triple point by a pair of a (white, positive) and a (black, negative) branch points, and a (white, negative) triple point by a pair of a (white, negative) and a (black, positive) branch points.

The effects of the smoothing are computed as follows. When a (black, negative) triple point is replaced by a pair of a (black, negative) and a (white, positive) branch points by a smoothing, the numbers $(T(B,-), P(B,-)$, $P(W,+))$ change to $(T(B,-)-1, P(B,-)+1, P(W,+)+1)$. This causes adding $x-z+(z-x)=0$ to $T P(G)$. Similarly the rest of the cases 
respectively cause addition of $-x+z-(z-x)=0, x+(z-x)-z=0$, and $-x-(z-x)+z=0$.

Therefore, we conclude that the expression does not change by smoothings. Let $G^{\prime}$ be the surface obtained from $G$ by smoothing all the triple points of $G$. Then $T P(G)=T P\left(G^{\prime}\right)$.

Since $G^{\prime}$ does not have any triple points, branch points are paired by embedded double point arcs. The neighborhood of these arcs are annuli since $G^{\prime}$ is orientable by construction. (Another possibility for non-orientable case is that the neighborhood can be a Mobius band.) Therefore each such pair consists of branch points of opposite signs and the same color. Thus for $G^{\prime}$ we have $P(B,+)=P(B,-)$ and $P(W,+)=P(W,-)$. Thus the expression for $G^{\prime}, T P\left(G^{\prime}\right)$, is zero.

In the above discussion we proved that for any $x$ and $z$ the expression $T P(G)=0$. Thus the coefficients of both $x$ and $z$ are 0 , and we obtain the identities.

Remark 6.2. Both these identities together imply the identity (4.2) but not vice versa.

Lemma 6.3. For an immersed diagram of a knotted surface, it holds that

$$
T(B,+)+T(W,+)=T(B,-)+T(W,-) .
$$

In other words, the number of positive triple points and that of negative ones are equal.

Proof. The double point curve $D$ is an oriented immersed closed curve in 3 -space for an immersed projection of a knotted surface. Push $D$ off of $f(F)$ towards the normal vector's direction to get an oriented immersed closed curve $D^{\prime}$ such that $D^{\prime}$ intersects $f(F)$ in finitely many isolated points. Thus $D^{\prime}$ lies in the region where two normals point into along the double point set of $f(F)$. The surface $f(F)$ and the curve $D^{\prime}$ has intersection points only at neighborhoods of triple points. Near a triple point, the self-intersection point of $D^{\prime}$ lies in the distinguished region. By Proposition 3.2 (2), the intersection number between $f(F)$ and $D^{\prime}$ is 1 (resp. -1 ) if the triple point is positive (resp. negative) where we define that the intersection is 1 (resp. -1) if an orientated curve intersects $f(F)$ in the same (resp. opposite) direction as the normal to $f(F)$. Since 3-space has trivial 1- and 2-dimensional integral homology, the intersection number of $f(F)$ and $D^{\prime}$ is zero. This proves the Lemma.

Theorem 6.4. For an immersed diagram of a knotted surface, it holds that

$$
T(B,+)=T(B,-), T(W,+)=T(W,-) .
$$

In other words, the number of positive triple point and that of negative ones are equal for each color.

Proof. This follows from the preceding Lemma and Theorem. 


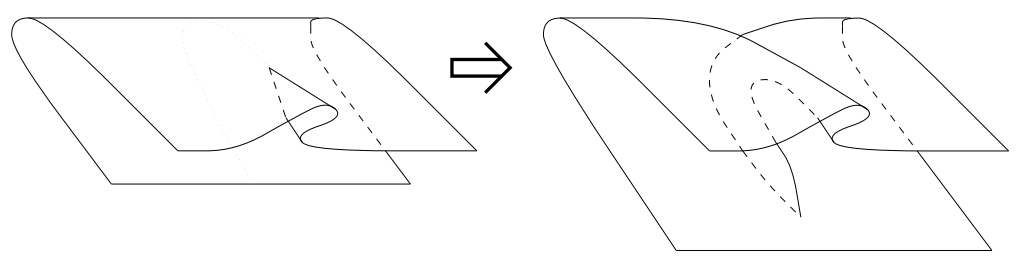

Figure 6. A cusp passing through a fold line.

\section{Cusps and Knotted Surfaces.}

\subsection{Canceling Cusps on Planar Projections of Knotted Surfaces.} We look at planar projections of knotted surfaces. Let $p: \mathbf{R}^{4} \rightarrow \mathbf{R}^{2}$ be a projection onto a 2-dimensional subspace which does not intersect with $f(F)$ and such that $\left.p\right|_{f(F)}$ is generic. Thus $(p \circ f)(F)$ has folds and cusps. Furthermore cusps are isolated and do not intersect with other folds, and folds have isolated transverse double crossing points. We prove that we can cancel pairs of cusps by isotopies of knotted surfaces in this section. The proof uses the projection $q_{1}: \mathbf{R}^{4} \rightarrow \mathbf{R}^{3}$ onto 3 -space to visualize the knotted surface.

We remark that Kamada's notion of surface braids certainly project without cusps, but these are not generic projections. A slight perturbation of the surface will result in a surface with at least one cusp in the neighborhood of the branch point. Furthermore, the proof for canceling cusps does not depend on the orientability of the surface. However, in proving that result, we have to introduce branch points that are generic singularities of maps from surfaces into 3 -space. In Section 7.3 we prove that orientable surfaces embedded in 4-space are isotopic to surfaces whose generic projections have neither cusps or folds. We mention here that planar projections played an essential role in the study of knotted surfaces in [5], and in [4] we used the planar diagram to show that the "one third turned trefoil" toroidal surface is unknotted. In this section we prove:

Proposition 7.2. Let $f: F \rightarrow \mathbf{R}^{4}$ be a knotted surface, and $p: \mathbf{R}^{4} \rightarrow \mathbf{R}^{2}$ be a projection onto a 2-dimensional subspace that does not intersect $f(F)$, and such that $\left.p\right|_{f(F)}$ is generic. Then $f$ is isotopic to $g$ such that $(p \circ g)(F)$ has none or a single cusp depending on whether the Euler characteristic of $F$ is even or odd respectively.

Proof. The parity of the cusp set is the same as the parity of the Euler characteristic [27]. There is a collection of embedded arcs on the surface that connects the cusps in pairs with the odd Euler characteristic case leaving an odd man out. These arcs may be chosen to be mutually disjoint, for at a transverse intersection between two arcs, the arcs can be re-routed via the 

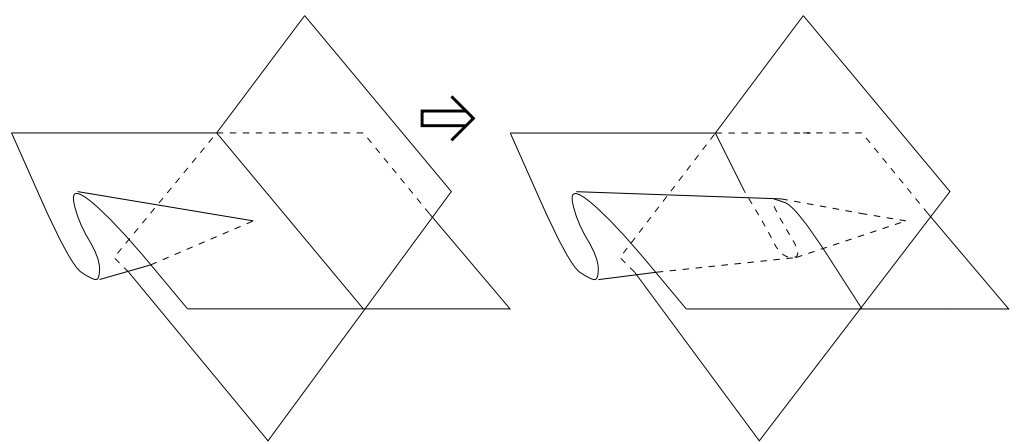

Figure 7. A Cusp passing through a sheet.
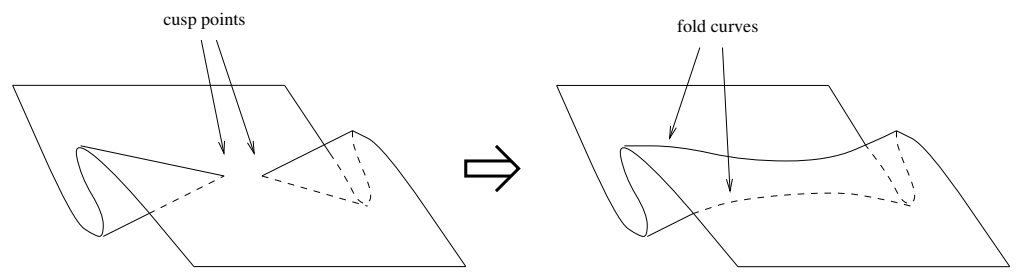

Figure 8. Canceling a pair of cusps.

local replacement $x y=0 \mapsto x y=-1$. Furthermore the arcs can be chosen to initially lead away from the cusps as indicated on the left of Fig. 6. The cusps are then pushed along the connecting arcs as indicated in Figs. 7 and 6 until a pair of cusps is joined by a short arc. If the cusps, appear as on the left of Fig. 8, then they can be canceled as on the right of that figure.

On the other hand, the two proximate cusps may be of differing types as indicated in Fig. 9. In this case we change the type of one of these as indicated in Fig. 10, and then apply the cancelation of Fig. 8. In Fig. 10, a pair of branch points has been introduced in the projection $q_{1}$. But the bottom of the figure indicates that surface is embedded in this neighborhood. It is an excercise in the moves from [5] to see that the resulting surface is ambiently isotopic to the original.

Cancel each pair of cusps that are joined by arcs in this fashion. In the odd Euler characteristic case only one cusp remains. In the even case, there are no more.

7.3. Canceling Cusps and Branch Points. While canceling a pair of cusps, it may be necessary to introduce a pair of branch points. On the other hand, surfaces that have trivial normal Euler class project without branch points $[6]$. For example, orientable surfaces satisfy this condition. In 

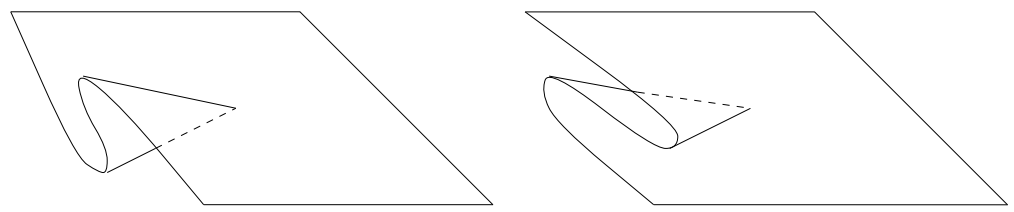

Figure 9. Two types of cusps.

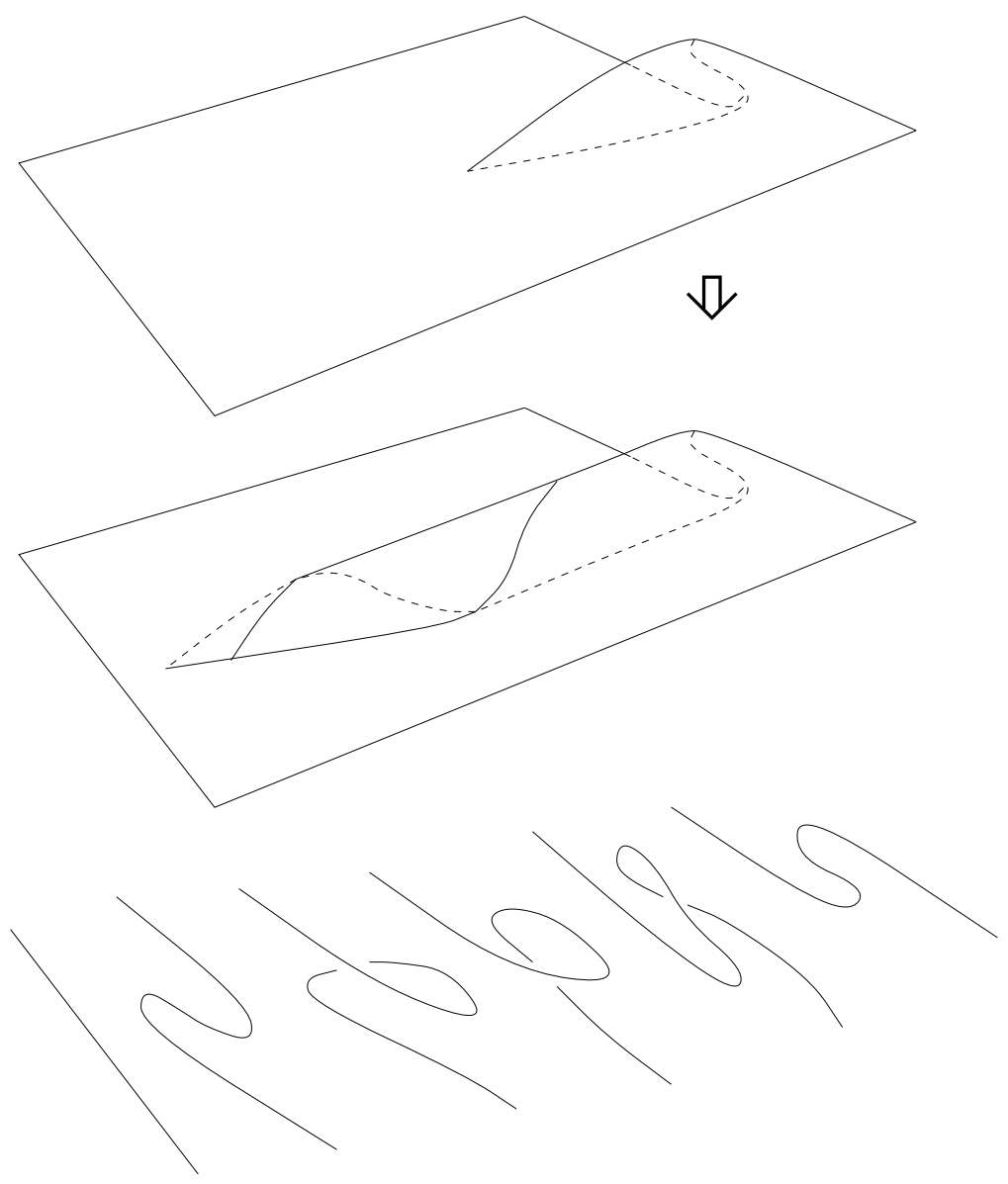

Figure 10. Changing types of cusps.

this section, we show that orientable surfaces are isotopic to surfaces that project with neither branch points nor cusps.

Theorem 7.3.1. Any orientable knotted surface can be isotoped so that the projection into 3-space has no branch points and the projection into a plane has no cusps. 

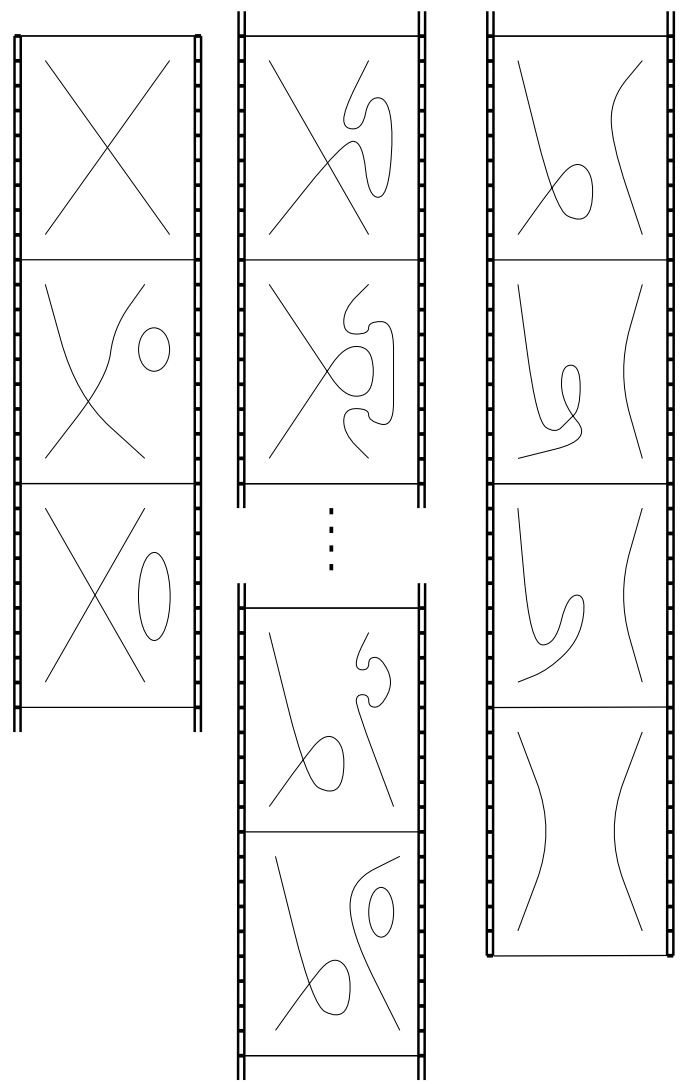

Figure 11. A generic perturbation of surface braids.

We use the theory of surface braids to prove this theorem.

7.4. Generic perturbations of surface braids. In the description of charts for surface braids, every branch point is mapped non-generically into the plane. There are several ways to get generic planar maps by perturbing the map. Here we use the generic map depicted in Fig. 11. It is seen that this generic map is isotopic to the original surface braid locally. Notice that there is only one cusp and one branch point in this perturbation. The pertubation is a specific choice of moving the surface slightly in 4-space so that its projection into the plane consist of the two small (dotted) circles of fold lines. The fold lines correspond to the critical points in the stills of the movie with the respect to the obvious height function on the page. The solid arc is the projection of the double curve; its end point is the branch point. Each still in the movie correspond to a non-critical slice of the chart on the right of the figure. Top to bottom on the still corresponds to right to left on the chart. For example, in the 4th still in the movie we see (reading from 
top to bottom) a maximum a minimum a subsequent maximum, a crossing point and a minimum. In the next still, a pair of maximum and minimum points are introduced by a saddle point move; these are the critical points on the left (therefore below in the still) of the image of the double curve.

Such a perturbation is chosen for each branch point of the chart. Notice then that the cusps that are introduced are all of the form $-x^{3}-t x$ for $t \in[-1,1]$.

Proof of Theorem 7.3.1. Any orientable knotted surface is isotopic to a closed surface braid. This gives a particular map $q_{1}: \mathbf{R}^{4} \rightarrow \mathbf{R}^{3}$ and $q_{2}: \mathbf{R}^{3} \rightarrow \mathbf{R}^{2}$.

Recall that the sign of a branch point is positive if the branch point appears as the birth of a positive braid generator or the death of a negative braid generator in the braid movie. Otherwise it is negative. Furthermore, the number of positive branch points is equal to the number of negative branch points since each branch point gives a local contribution to the normal Euler class, and that is a trivial invariant for orientable surfaces $[\mathbf{6}, \mathbf{1}]$.

For $i=1, \ldots n$, let $\gamma_{i}$ be an arc on the surface braid $F$ that connects a pair branch points of opposite signs. Assume that the $\operatorname{arcs} \gamma_{i}$ are in general position with double decker curves and each other. This means that the arcs intersect the pre-image of the double curves and intersect each other in a finite number of transverse points. (We assume that $F$ is connected here, however, for disconnected case the argument can be repeated componentwise.) We can also assume that the $\operatorname{arcs} \gamma_{i}$ stay inside the smallest fold circle of the closed surface braid.

If a pair of arcs, say $\gamma_{i}$ and $\gamma_{j}$ intersect, then we can smooth this intersection to get two new arcs that each connect a pair of positive and negative branch points. By induction on the number of intersection points, we may assume then that the connecting arcs do not intersect.

By isotopy perturb the map locally at branch points so that we get a generic planar map such that at each branch point the map is as described in the preceding section.

Each cusp is near an associated branch point. So we push the cusps along the arcs that joint them as in the proof of Prop. 7.2. The branch points follow close behind. These arcs are pairwise disjoint and only intersect the fold set at their end points.

On the other hand, the arc on the surface that joins the pair of cusps might intersect the double decker set of the projection. In this case the branch point and cusp can be pushed through the double point set.

Therefore the cusps and branch points are pushed to be near each other along their joining arcs. They can be canceled without using a Whitney trick (such as was needed in [6]). One of the cusps is changed as depicted in 


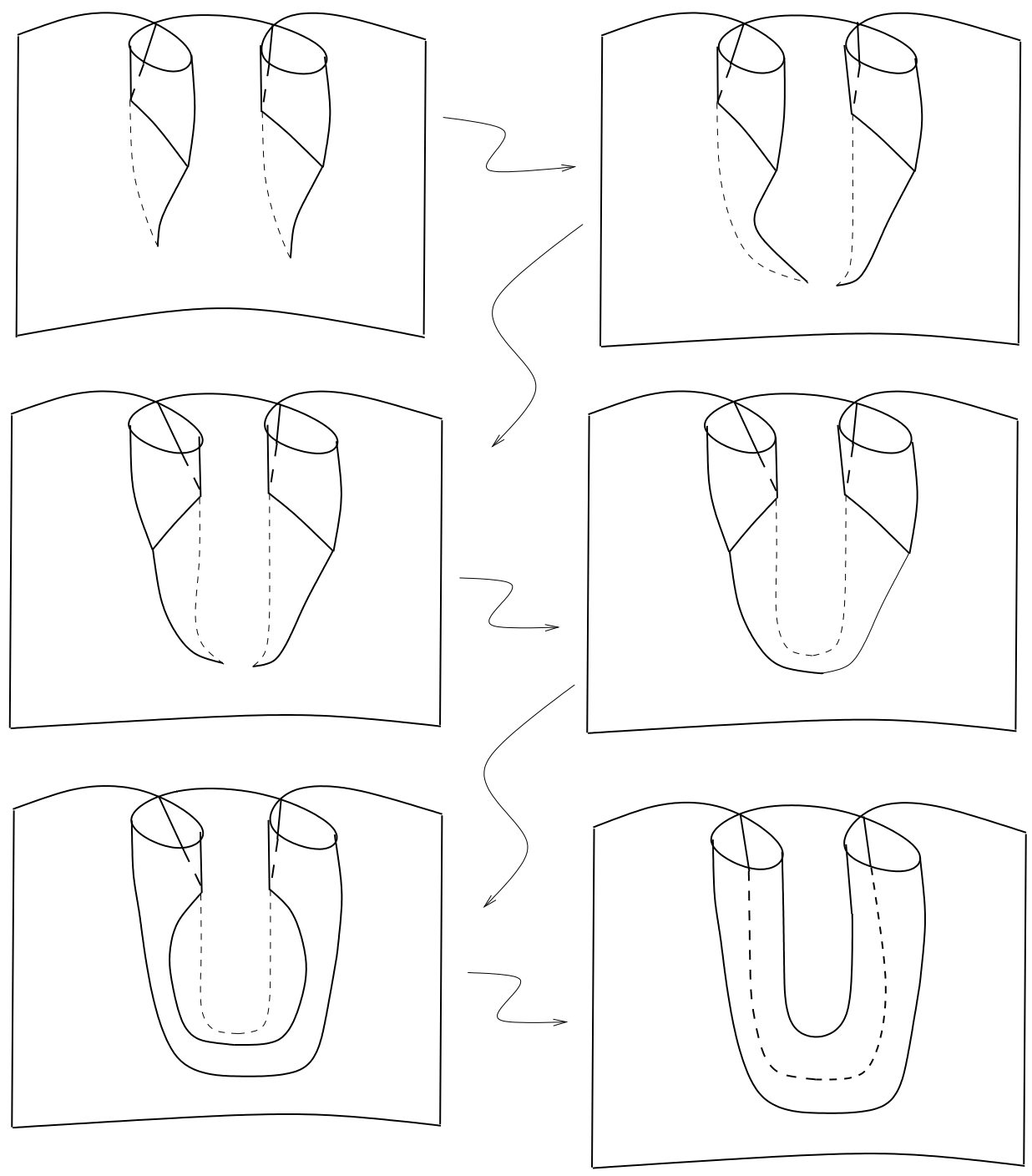

Figure 12. Canceling both cusps and branch points.

Fig. 12. In particular, no new pairs of branch points need to be introduced. This completes the proof.

We conclude the paper with a conjecture.

Conjecture 7.4.1. Any knotted surface can be isotoped so that the projection into a plane has no cusps or a single cusp depending on the Euler characteristic is even or odd respectively, and the projection into 3-space has the same number of branch points as the absolute value of twice the normal 
Euler number. In other words we can minimize both the numbers of cusps and branch points by isotopy.

\section{References}

[1] T.F. Banchoff, Double tangency theorems for pairs of submanifolds, in 'Geometry Symposium', Utrecht 1980, eds. Looijenga, Seirsma, and Takens, LNM, 894, Springer-Verlag, (1981), 26-48, MR 83h:53005, Zbl 474.53007.

[2] J. Birman and X.S. Lin, Knot polynomials and Vassiliev's invariants, Invent. Math., 111(2) (1993), 225-270, MR 94d:57010, Zbl 812.57011.

[3] J.S. Carter, D.E. Flath and M. Saito, The Classical and Quantum 6j-symbols, Princeton University Press, Lecture Notes in Math Series, 1995, MR 97g:17008.

[4] J.S. Carter, L.H. Kauffman and M. Saito, Diagrammatics, singularities, and their algebraic interpretations, Matemática Contemporânea, 13 (1997), 10th Brazilian Tolopogy Meeting, Sociedade Brasileira de Matemática (Rio de Janeiro, 1997), 21-115, MR 99d:57016, Zbl 930.57001.

[5] J.S. Carter, J.H. Rieger and M. Saito, A diagrammatic description of knotted surfaces and their isotopies, Advances in Mathematics, 127(1) (April 15, 1997), 1-51, MR 98c:57023, Zbl 870.57032.

[6] J.S. Carter and M. Saito, Canceling branch points on the projections of surfaces in 4-space, Proc. A.M.S., 116(1) (1992), 229-237, MR 3i:57029.

$[7]$ , Reidemeister moves for surface isotopies and their interpretation as moves to movies, J. of Knot Theory and its Ram., 2(3) (1993), 251-284, MR 94i:57007, Zbl 808.507020.

[8] _ Braids and movies, Journal of Knot Theory and Its Ramifications, 5(5) (1996), 589-608, MR 97j:57028.

[9] _ Knotted surfaces, braid movies and beyond, in J. Baez, "Knots and Quantum Gravity," Oxford Science Publishing (Oxford 1994), 191-229, Zbl 859.57027.

[10] _ Knot diagrams and braid theories in dimension 4, The Proceedings of the 3rd International Conference on Real and Complex Singularities, ed. Marar, 112-147, MR 98g:57037, Zbl 849.57022.

[11] _ A diagrammatic theory of knotted surfaces, in 'Quantum Topology', eds. R. A. Baadhio and L. H. Kauffman, World Scientific, (1993), 91-115, MR 95c:57041, Zbl 838.57015.

[12] _ A Seifert algorithm for knotted surfaces, Topology, 36(1) (1997), 179-201, MR 97f:57026, Zbl 865.57022.

[13] Normal Euler classes of knotted surfaces and triple points on projections, Proc. A.M.S., 125(2) (1997), 617-623, MR 97d:57030, Zbl 871.57024.

[14] Surfaces in 3-space that do not lift to embeddings in 4-space, in 'Banach Center Proceedings', 42 (1998) Knot Theory, 29-47, MR 9h:57047, Zbl 910.57009.

[15] _ Knotted Surfaces and Their Diagrams, American Mathematical Society, Surveys and Monographs, 55 (Providence, 1998), MR 98m:57027, Zbl 904.57010.

[16] J.H. Conway, An enumeration of knots and links and some of their algebraic properties, in 'Computational Problems in Abstract Algebra', Pergamon Press, New York, (1970), 329-358, MR 41 \#2661, Zbl 202.54703. 
[17] C. Giller, Towards a classical knot theory for surfaces in $\mathbf{R}^{4}$, Illinois Journal of Mathematics, 26(4) (Winter 1982), 591-631, MR 84c:57011, Zbl 485.57013.

[18] M. Golubitsky and V. Guillemin, Stable Mappings and Their Singularities, GTM, 14, Springer Verlag, (1973), MR 49 \#6269, Zbl 294.58004.

[19] V.F.R. Jones, Hecke algebra representations of braid groups and link polynomials, Ann. of Math., 126 (1987), 335-388, MR 89c:46092, Zbl 631.57005.

[20] S. Kamada, Surfaces in $R^{4}$ of braid index three are ribbon, Journal of Knot Theory and its Ramifications, 1 (1992), 137-160, MR 93h:57039, Zbl 763.57013.

[21] _ Alexander's and Markov's theorems in dimension four, Bull. A.M.S., 31 (1994), 64-67, MR 94j:57023, Zbl 831.57013.

[22] _ A characterization of groups of closed orientable surfaces in 4-space, Topology, 33 (1994), 113-122, MR 95a:57002, Zbl 820.57017.

[23] L.H. Kauffman, Formal Knot Theory, Princeton U. Press, Mathematical Notes, 30 (1983), MR 85b:57006, Zbl 537.57002.

[24] L.H. Kauffman, Knots and Physics, World Scientific, Series on knots and everything, 1 (1991), MR 93b:57010, Zbl 733.57004.

[25] L.H. Kauffman and S. Lins, Temperley-Lieb Recoupling Theory and Invariants of 3manifolds, Ann. of Math. Studies, no. 134, Princeton U. Press, Princeton, NJ, 1994, MR 95c:57027, Zbl 821.57003.

[26] H.I. Levine, Elimination of cusps, Topology, 3 (1965), 263-295, MR 31 \#756, Zbl 146.20001.

[27] K.C. Millett, Generic smooth maps of surfaces, Top. and its Appl., 18 (1984), 197215, MR 86j:57014, Zbl 554.57018.

[28] L. Rudolph, Braided surfaces and Seifert ribbons for closed braids, Comm. Math. Helv., 58 (1983), 592-599, MR 84j:57006, Zbl 522.57017.

[29] V. Vassiliev, Cohomology of knot spaces, in 'Theory of Singularities and its Applications', (V.I. Arnold, ed.), AMS (1990), 23-69, MR 92a:57016, Zbl 727.57008.

Received December 1, 1997 and revised October 30, 1998.

\author{
UnVERsidade de SÃo Paulo \\ InStituto DE MATEMÁtica \\ SÃo Paulo SP \\ BRASIL \\ E-mail address: vera@ime.usp.br \\ University of South Alabama \\ Mobile, AL 36688 \\ E-mail address: carter@mathstat.usouthal.edu \\ UNIVERSITY OF SOUTH FLORIDA \\ TAMPA, FL 33620 \\ E-mail address: saito@math.usf.edu
}

